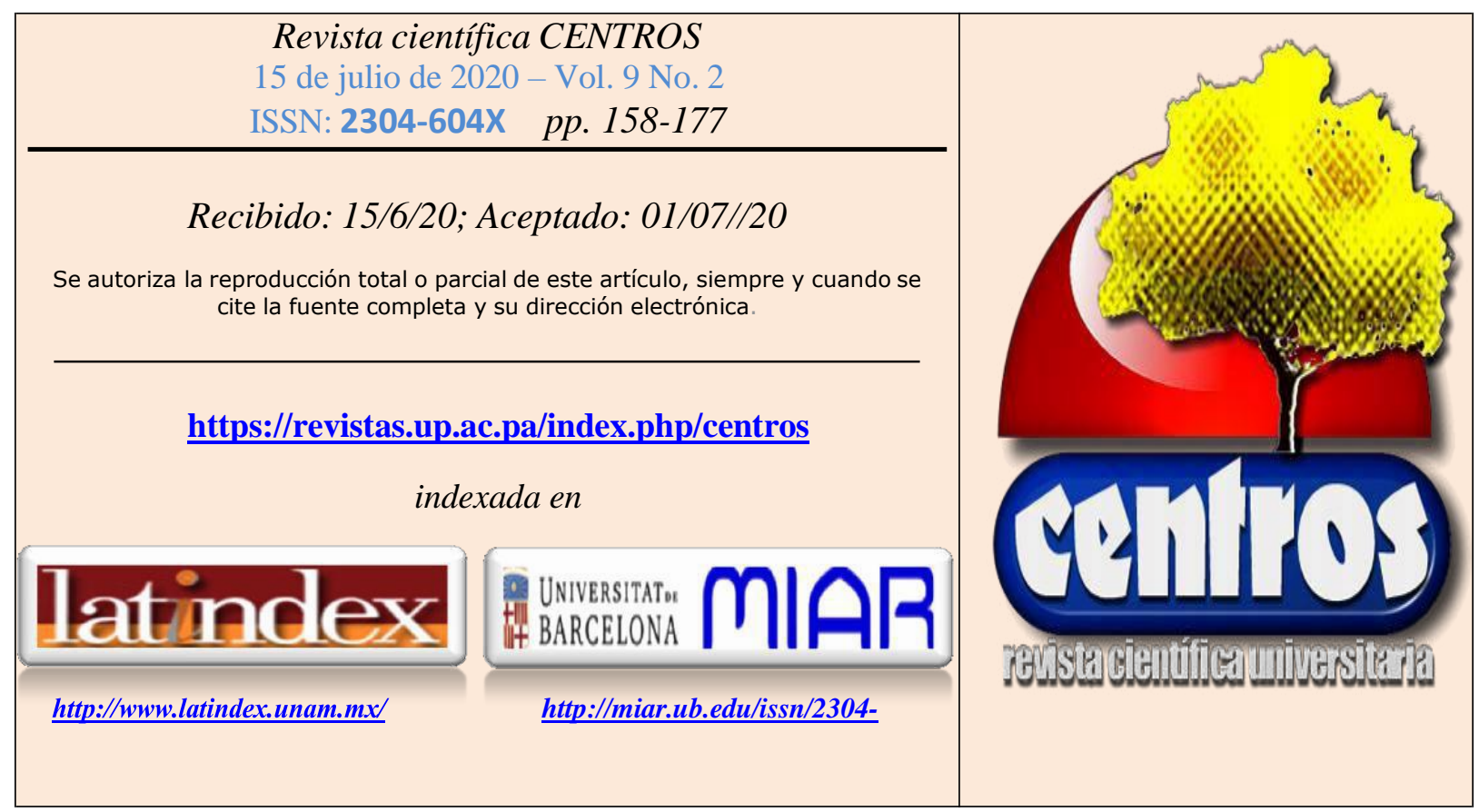

\title{
Zona Libre de Colón: Consideraciones para expandir su actividad comercial e impulsar su competitividad post COVID-19
}

Colon Free Zone: Considerations toward expanding its commercial activity and enhancing competitiveness post COVID-19

\section{Carlos Manuel Gómez-Rudy ${ }^{1}$}

'Universidad de Panamá, Centro Regional Universitario de Colón, Facultad de Administración de Empresas y Contabilidad, E-mail: carlos.gomezr@up.ac.pa. ORCID: 0000-0003-4118-4259

\section{Resumen}

La Zona Libre de Colón es un componente crítico del sistema logístico y la economía de Panamá, y funge como plataforma de redistribución de mercancías a nivel de la región. Debido a la pandemia del COVID-19 originada en China, las cadenas de suministros han sufrido disrupciones debido al cierre de fábricas en las fuentes de abastecimiento de Asia, Norteamérica y Europa. Esta situación ha afectado los flujos de mercancías hacia la zona franca aunado a la contracción de la demanda de los mercados regionales de Centroamérica, Suramérica y el Caribe, lo que sugiere examinar el modelo de funcionamiento vigente con el fin identificar los ajustes 
necesarios ante la nueva dinámica del mercado. El estudio es descriptivo, de tipo no experimental y longitudinal, examinando el comportamiento del movimiento comercial histórico y reciente. Los resultados muestran una disminución de $40.2 \%$ en el valor anual de las transacciones entre 2012 a 2019, y un $40.9 \%$ en el primer cuatrimestre de 2020. Ante el deterioro de la actividad comercial, se propone un nuevo modelo de abastecimiento cercano (near-sourcing) complementado con cambios normativos, institucionales y procedimentales bajo un fuerte componente tecnológico, de digitalización y flexibilidad comercial, con el objetivo de modernizar la Zona Libre de Colón, expandir su actividad comercial e impulsar su competitividad.

\section{Palabras Claves}

Economía, logística, comercio internacional, distribución, inversión pública

\section{Abstract}

The Colon Free Zone is a critical component of the logistics system and the economy of Panama, and serves as a platform for the redistribution of goods at a regional level. Due to the COVID-19 pandemic originated in China, supply chains have suffered disruptions due to the closure of factories at supply sources in Asia, North America, and Europe. This situation has affected the flow of goods to the free zone, together with the contraction in demand from the regional markets of Central America, South America and the Caribbean, which suggests examining the current operating model in order to identify the necessary adjustments to the new market dynamics. The study is descriptive, non-experimental, and longitudinal, examining the behavior of the recent and historical trade movement. The results show a decrease of $40.2 \%$ in the annual value of transactions between 2012 to 2019 , and $40.9 \%$ in the first quarter of 2020. Given the deterioration of the commercial activity, a new model of nearby supply (near-sourcing) is proposed complemented by changes in regulations, institutions and processes under a strong technological component, digitalization and commercial flexibility, with the objective of modernizing the Colon Free Zone, expanding its commercial activity and enhancing competitiveness.

\section{Keyword}

Economics, logistics, international trade, distribution, public investment 


\section{Introducción}

A finales del 2019 en la ciudad de Wuhan, provincia de Hubei, China surge un brote de neumonía con características desconocidas. El 7 de enero de 2020, las autoridades de salud de ese país confirmaron la identificación de un nuevo coronavirus (COVID-19) (Zhang et al., 2020). La Organización Mundial de la Salud (OMS) declara el 30 de enero una emergencia internacional de salud pública capaz de expandirse a otros países (OMS, 2020a). Brasil reportó el 26 de febrero el primer caso de esta enfermedad en Latinoamérica (BBCMundo, 2020) y el 11 de marzo, la OMS (OMS, 2020b) anuncia la categoría de pandemia con más de 118,000 casos confirmados de COVID-19 a nivel mundial. Al 1 de junio, se han registrado un número 3,055,070 personas contagiadas y 377,428 fallecidos globalmente (Worldometers, 2020).

Este nuevo panorama de salud pública generó dos importantes fenómenos a nivel de la economía global, y consecuentemente en el comercio internacional: un shock de la oferta y un shock de la demanda. Debido a la suspensión de las actividades productivas de los principales centros de producción en China y el resto de Asia, y que rápidamente se extendió a Europa y Norteamérica, el cierre de fronteras y las medidas de cuarentena, se redujo la producción global y no se pudo cumplir con las cadenas de suministros.

Por el lado de la demanda, se refleja una contracción del consumo global derivado de las restricciones de movilidad de la población, el progresivo efecto en la suspensión laboral y la pérdida de fuentes de ingresos de un número significativo de la población (BID, 2020a). Aunado al consecuente impacto en la disminución de los precios y la quiebra de muchas empresas, los gobiernos se verán afectados en su recaudación fiscal y balances presupuestarios (Clavellina \& Domínguez, 2020)

Este particular fenómeno iniciado en Wuhan (China) impactó progresivamente en las exportaciones asiáticas reduciendo drásticamente la disponibilidad de bienes de todo tipo a nivel mundial. La pandemia representa una de las mayores disrupciones 
encontradas durante las últimas décadas provocando el deterioro de muchas de las cadenas de suministros globales (Araz et al., 2020).

Un brote epidémico como el COVID-19 representa un caso especial de riesgo en las cadenas de suministros caracterizado por tres componentes distintivos: (i) disrupciones a largo plazo y su impredecible escalada, (ii) la simultánea expansión de las rupturas en la cadena de suministro y la propagación de los contagios en la población, y (iii) la simultánea disrupción en la oferta, demanda e infraestructura logística (Ivanov, 2020).

Debido al COVID-19, Latinoamérica no escapa a las afectaciones de las cadenas de suministros globales. Los efectos de la pandemia impactan a la región en medio de una situación de alta fragilidad. Previo al reporte de la enfermedad, las exportaciones se contrajeron $2.2 \%$ en el promedio anual de 2019, mientras que el intercambio global se redujo en $2.8 \%$. En ambos casos, la disminución se debió a la merma de los precios y al estancamiento de los volúmenes exportados. Se estima que el valor total de las ventas externas de la región disminuyó un 3.2\% para el primer trimestre de 2020 (BID, 2020b).

Este panorama de una débil economía regional ha influido negativamente en el desempeño económico de Panamá, e incide directamente a la Zona Libre de Colón (ZLC), que es un componente crítico del sistema logístico panameño y cumple un rol fundamental como plataforma de redistribución de mercancías a nivel de la región. La ZLC representa uno de los clústeres de actividad comercial más importante del país dedicado principalmente a la importación y reexportación al por mayor de bienes finales y semielaborados bajo un marco de beneficios fiscales, así como ofrecer servicios complementarios de transporte, intermediación logística, valor agregado y financieros, entre otros (GTP, 2020; Slack \& Gouvernal, 2016).

Esta zona franca representó el $4.2 \%$ del producto interno bruto de Panamá en el año 2018 (CNC, 2019) y como motor de la economía por las actividades de comercio exterior, genera aproximadamente 20,000 empleos directos e indirectos, con aportes al Estado que superan los B/.15 millones al año (Forbes Centroamérica, 2020). 
Ante la fuerte contracción de los mercados regionales durante los últimos años y los shocks económicos agudizados por el COVID-19, surge la pregunta: ¿Debe la Zona Libre de Colón plantear nuevas estrategias ante los cambios dinámicos de los mercados internacionales?

El propósito del estudio es describir los flujos comerciales recientes de esta zona franca bajo el modelo comercial vigente y examinar si existe la necesidad de realizar ajustes hacia un nuevo modelo de funcionamiento que permita expandir su actividad comercial e impulsar su competitividad.

\section{Material y Método}

\section{Tipo de investigación}

La investigación es descriptiva, de tipo no experimental y de corte longitudinal dado que tiene como propósito basarse fundamentalmente en la obtención de conclusiones sobre el estado del arte de la Zona Libre de Colón y las oportunidades para replantear un nuevo modelo de funcionamiento luego del COVID-19. Su carácter descriptivo obedece a una revisión estadística sobre el movimiento comercial reciente y el efectivo desempeño del modelo operativo existente. Es no experimental ya que las variables del fenómeno no serán manipuladas, sino examinadas en su propio entorno, mientras que el enfoque longitudinal corresponde a un tratamiento de los datos a lo largo del tiempo.

Para el desarrollo de la investigación se han considerado las etapas del proceso: recolección y análisis de datos. Los datos estadísticos serán analizados descriptivamente de manera longitudinal con el fin de establecer patrones históricos de comportamientos que permitan identificar proporciones y magnitudes con respecto a las diferentes variables examinadas. 
Las fuentes para los datos estadísticos son los informes oficiales que emite el Departamento de Estadística y Estudios Económicos de la ZLC, y los anuarios de comercio exterior del Instituto de Estadística y Censos de la Contraloría General de la República. El estudio tiene como alcance el desempeño de las actividades comerciales de la ZLC entre los años 2012 a 2019, y el primer cuatrimestre del año 2020. Los datos son presentados en tablas que permitan comparar el desempeño anual de algunos países seleccionados, y en gráficos para mostrar el comportamiento de las variables a lo largo de los años en estudio.

\section{Resultados}

\section{Sobre el modelo comercial vigente de la Zona Libre de Colón}

Las actividades fundamentales de importación y reexportación poseen un espectro con alcance regional. La posición geo-estratégica del país le ofrece a la ZLC una alta conectividad entre los principales proveedores originarios de Asia, Europa y América del Norte, de los cuales se importa un amplio portafolio de productos que se distribuyen hacia toda América Central, el Caribe y gran parte de los países de América del Sur.

El modelo comercial vigente posee una directa relación entre los países productores (países de importación), los mercados regionales de consumo (países de reexportación) y los bienes comercializados. Para la ejecución de estas actividades, la operación de la ZLC como centro de distribución supone la existencia de dos grandes nodos: los centros de producción y los mercados de consumo (Figura 1). Los primeros representan las fuentes de todos sus insumos de producción o comercialización originados en otros países, ya sean materias primas, bienes semielaborados o bienes finales; mientras, los mercados de consumo representan los clientes finales en terceros países, ya sean importadores, comercializadores o distribuidores finales (Gómez-Rudy \& Ortega-Gómez, 2016). 


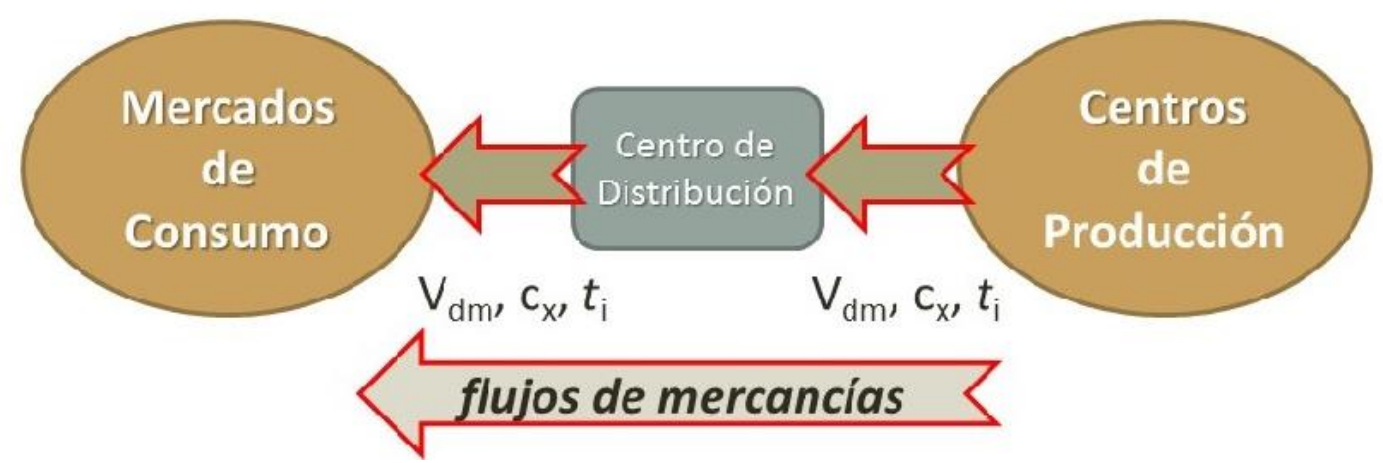

Figura 1. Modelo de distribución de la Zona Libre de Colón. Fuente: GómezRudy \& Ortega-Gomez (2016)

Para que la ZLC logre una sostenibilidad y mantener la capacidad competitiva frente a otros centros de operaciones logísticas en países vecinos, los flujos de mercancías deben considerar tres parámetros altamente valorados: los volúmenes de productos demandados $\left(V_{d m}\right)$, los costos de las mercancías y comercialización $\left(C_{x}\right)$ y el tiempo de traslado del inventario comercializado ( $\left.t_{i}\right)(\mathrm{lbid})$.

Según datos oficiales de la ZLC, el movimiento comercial acumulado anual de esta zona fue B/.8,712 millones en 2003 y alcanzó su punto máximo histórico en 2012 con B/.30,793 millones (CGR, 2019). A partir del 2013, se evidencia una continua contracción comercial debido a varios fenómenos: la devaluación del yuan (China), las restricciones comerciales de Colombia, del deterioro del mercado de Venezuela y la ralentización de los mercados de Ecuador, Centroamérica y el Caribe. Como se muestra en la Figura 2, es evidente la disminución (-40.2\%) de su actividad comercial entre 2012 a 2019, cerrando este último año con B/.18, 469 millones. 


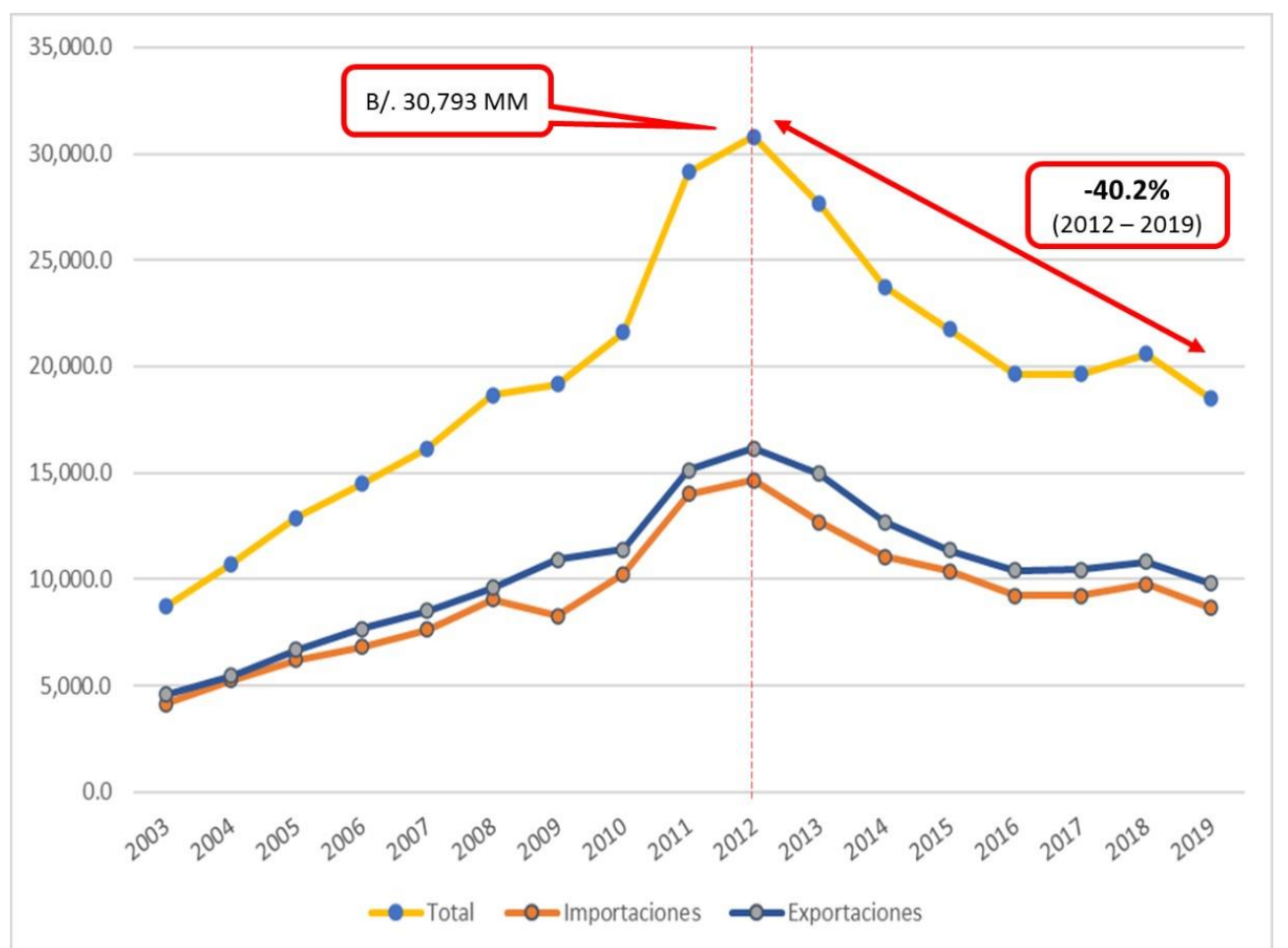

Fuente: CGR (2019), ZLC (2020b, 2019)

Figura 2. Movimiento comercial de la Zona Libre de Colón. Años: 2003 - 2019 (en millones de balboas)

Los principales flujos comerciales de países proveedores de la ZLC (China, Estados Unidos, Singapur, Hong Kong y México) han mostrado fuertes contracciones al comparar el valor acumulado de las transacciones durante los años 2012, 2015, 2018 y, los resultados finales de 2019. La Figura 3 muestra las principales fuentes de estas importaciones y cómo ha disminuido el flujo de mercancías en términos de millones de balboas.

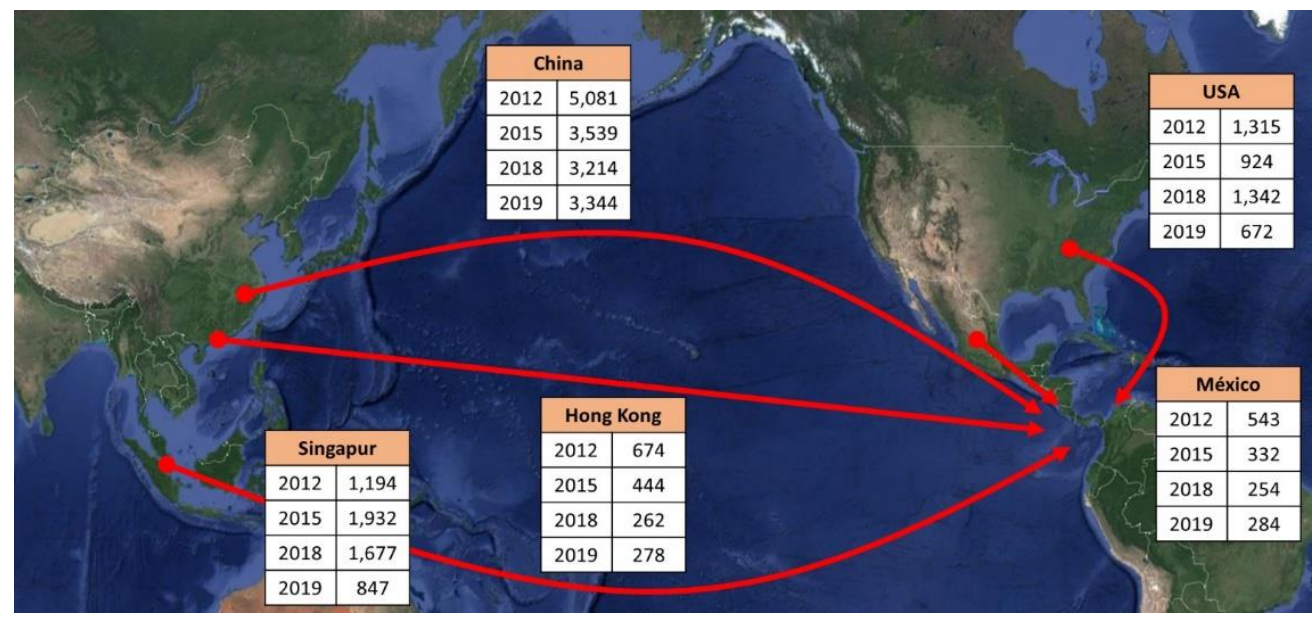

Figura 3. Principales flujos comerciales de importación de la ZLC. Años: 2012, 2015, 2018 y 2019 (en millones de balboas)

Fuente: CGR (2019), ZLC (2020b, 2019) 
Desde la ZLC, se reexportan mercancías hacia diversos países de Latinoamérica. En casi todos los mercados se evidencia una reducción de las ventas dadas en millones de balboas. Se destaca cómo disminuye la tasa de crecimiento de sus principales mercados entre 2012 a 2019: Puerto Rico (-79.5\%), Venezuela (86.3\%), Colombia (-54.4\%) y Ecuador (-19.3\%). Panamá también representa un mercado significativo para la ZLC. Al ser una zona franca, su actividad es considerada foránea y libre de impuestos, por tanto, toda venta a cualquier nacional o empresa del país requiere el pago de los impuestos de importación. Sin embargo, aún con una reducción del $28.1 \%$, ahora Panamá representa el principal cliente de la ZLC con ventas de B/. 969.0 millones (Figura 4).

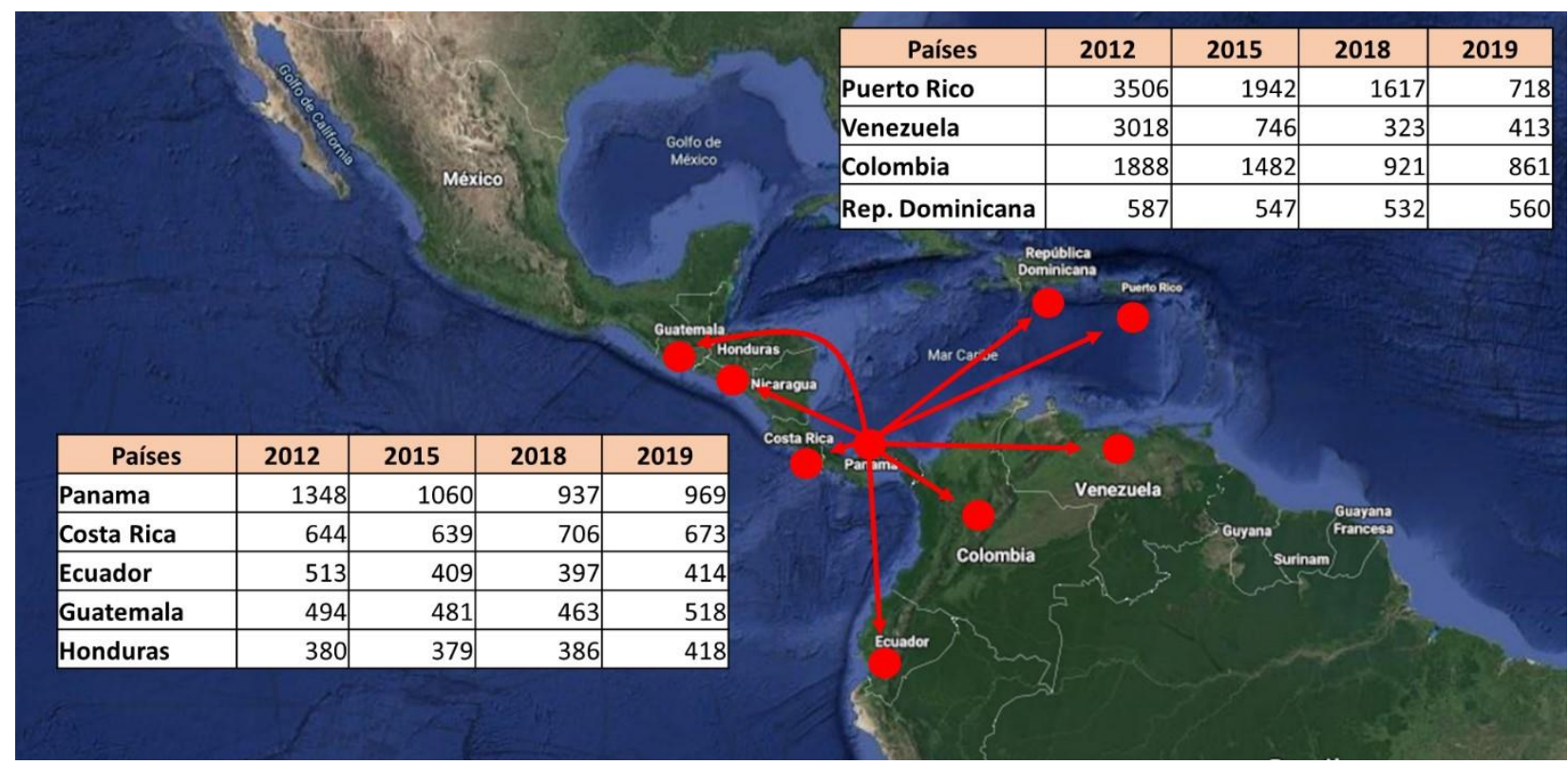

Fuente: CGR (2019), ZLC (2020b, 2019)

Figura 4. Principales flujos comerciales de exportación de la ZLC. Años: 2012, 2015, 2018 y 2019 (en millones de balboas)

A pesar de existir una fuerte disminución en la actividad comercial de la ZLC en los últimos 7 años, la Figura 5 muestra cómo el COVID-19 ha impactado fuertemente en la dinámica de comercio exterior al comparar el primer cuatrimestre del 2019 con el mismo período 2020. Entre enero a abril de 2020, las contracciones de la demanda regional ha influido a tales niveles que se alcanzó una disminución del 40.9\%, finalizando en el mes de abril con un movimiento comercial de B/.789.6 millones, entre importaciones (B/.447.8 millones) y reexportaciones (B/.341.8 millones). 


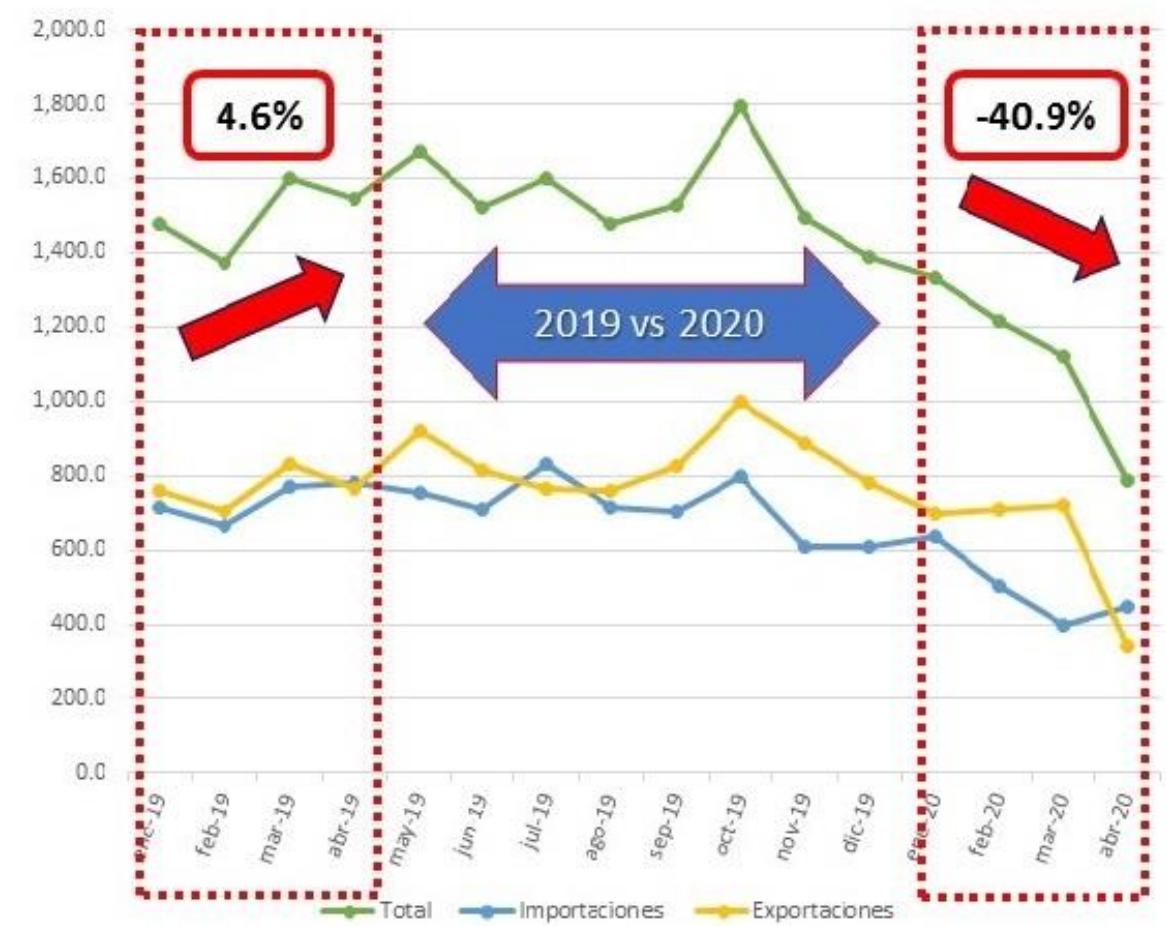

Fuente: ZLC (2020a, 2020b)

Figura 5. Movimiento comercial mensual de la ZLC. Años: $2019-2020$ (en millones de balboas)

Los resultados anteriores muestran una radiografía de cómo la actividad de comercio exterior realizada en la ZLC ha decrecido tanto en las importaciones, desde sus principales países proveedores (centros de producción), como en las reexportaciones, sus principales países compradores (mercados de consumo). Los efectos de esta contracción redundarán en las aproximadamente 700 empresas que están registradas en la zona, con significativos impactos en los más de 39,400 empleos directos, indirectos e informales, y su equivalente en salarios y otros ingresos mensuales por B/.25 millones, según estimaciones realizadas a inicios de la pandemia de COVID-19 (Gómez-Rudy, 2020).

\section{Sobre un nuevo modelo para la Zona Libre de Colón}

El diagnóstico del movimiento comercial de la ZLC bajo el modelo de distribución vigente sugiere la necesidad de examinar nuevas propuestas que permitan expandir las reexportaciones e impulsar su competitividad. Ante el COVID19, los grandes centros de producción, principalmente de Asia, han generado disrupciones en las cadenas de suministros hacia los mercados de todo el mundo. Las 
dificultades de este modelo mediante el abastecimiento desde una fuente distante (distant sourcing) donde los costos operacionales (principalmente laborales) son más bajos, pero con una lenta capacidad de respuesta a los clientes indica que, ante el nuevo panorama global, debe replantearse la estructura y procesos de producción y distribución de la ZLC a un modelo más eficiente.

Reconociendo el valor del posicionamiento geoestratégico del país, la propuesta es ampliar la capacidad productiva de la ZLC hacia un centro de transformación, generación de valor agregado y distribución mediante diversas actividades que permitan convertir insumos, materiales y piezas en bienes finales, aprovechando las capacidades instaladas ociosas que actualmente existen o las capacidades disponibles para futura expansión.

La implementación de un modelo de abastecimiento cercano (near-sourcing) posibilita que los centros de producción alimenten de todos los componentes necesarios a las actividades de transformación y generación de valor en esta zona, y que desde este punto se realice la distribución más eficiente a los mercados de consumo y facilite los cambios dinámicos en las características de los productos y las preferencias de los clientes.

La Figura 6 muestra cómo cambiaría el modelo de distribución vigente de abastecimiento desde la fuente hacia un nuevo modelo de abastecimiento cercano.

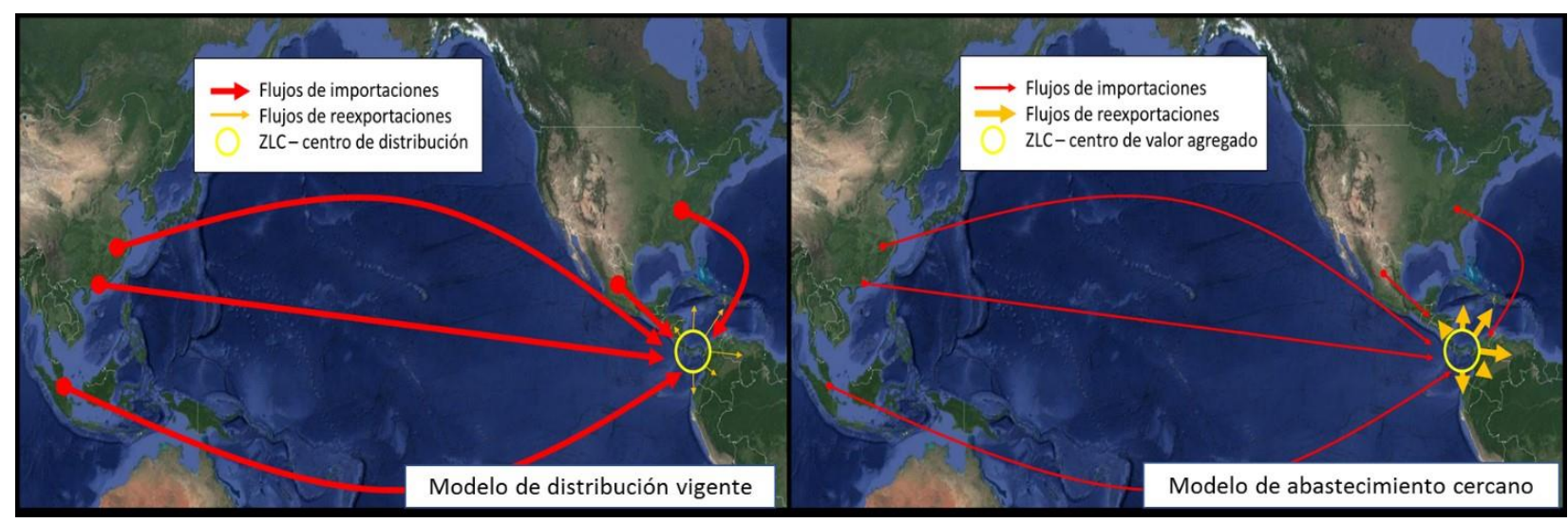


Figura 6. Comparación del comportamiento de los flujos comerciales de la ZLC en los modelos de distribución vigente (source at origen) y modelo de abastecimiento cercano (near-sourcing)

El modelo propuesto de abastecimiento cercano (near-sourcing) reactivaría un importante número de empresas que realizarían un amplio grupo actividades logísticas de valor agregado tales como el manejo de inventarios (inventory management), almacenamiento (warehousing), embarque (shipping), empaquetado (packing), etiquetado (labeling), armado de paquetes (kitting), reacondicionamiento de productos (product reconditioning), personalización (customizing), y logística inversa (reverse logistics), entre otras.

Con la incorporación de herramientas basadas en alta tecnología alineadas a la Logística 4.0, este modelo daría oportunidad al surgimiento de otro grupo de actividades de valor agregado pero con un enfoque más estratégico, como el análisis masivo de datos (big data), análisis predictivos del movimiento de la mercancía (predictive analysis), la visibilidad y trazabilidad de la mercancía mediante sistemas detorres de control (control towers) que ofrecen la posibilidad de un monitoreo activo a lo largo de las cadenas de suministros globales, posicionando a la ZLC como punto focal para estos nuevos desarrollos.

El cambio hacia una estrategia que fomente una producción cercana a los clientes y poder responder a sus intereses con mayor capacidad y flexibilidad en el producto, se impulsa por las ventajas competitivas del Hub Logístico de Panamá destacadas por el valor de la ruta y conectividad multimodal, las infraestructuras de calidad global (canal, puertos, aeropuertos), y el ámbito de atracción de inversiones logísticas de gran complejidad y oportunidades (Gabinete Logístico, 2019; Flórez et al., 2018).

Sin embargo, existen serias desventajas que restan capacidad competitiva al sistema como la reducida dimensión del mercado local, el incipiente grado de madurez 
del clúster logístico tanto institucional como empresarial, el bajo nivel de integración de los procesos, altos costos operativos, y el rezago en la digitalización e integración de tecnologías (Ibíd).

Dentro de la Estrategia Logística Nacional de Panamá 2030 (ELNP) se resalta la importancia crítica de la ZLC dentro del sistema logístico panameño, pero a su vez se destaca una crisis de inadaptación al nuevo marco de comercio internacional y la necesidad de una reorientación de su modelo funcional y de negocios a fin de evitar la pérdida de competitividad (Ibíd). Los cambios en el modelo de distribución y las exigencias del comercio internacional requiere de ajustes significativos en la forma y mecanismos de hacer negocios de las empresas de la ZLC (Sokol, 2017).

Desde la creación de la ZLC en 1948, su modelo fundamentado en la recepción, almacenamiento, venta y despacho de mercancías sin mayor valor agregado permitió generar un significativo ritmo comercial. Las bondades de localización geográfica, conectividad de transporte, facilidades financieras y conocimiento de los mercados mitigaron las barreras que tenían otros países de Latinoamérica logrando un nicho de negocios en la región. Sin embargo, este modelo ha evidenciado fuertes niveles de agotamiento demostrado con la continua contracción de sus flujos comerciales hasta la fecha, tal como se señaló en la Figura 2.

Al analizar las oportunidades de un nuevo modelo basado en el abastecimiento cercano (near-sourcing) que impulsaría el movimiento de mercancías, y se fortalece con actividades logísticas y estratégicas de valor agregado, es imprescindible realizar importantes cambios que modernicen la ZLC. Estos cambios deben incluir (i) una imagen corporativa e institucional más dinámica y enfocada a la apertura global, (ii) la actualización pertinente y continua de la normativa que rige la ZLC, (iii) una facilitación institucional efectiva, (iv) la equiparación de los regímenes fiscales, migratorios y laborales entre todas las zonas económicas especiales, (v) impulsar la figura del comercio electrónico, (vi) la apertura a mecanismos de documentación y pago digitales, (vii) mejoras de las infraestructuras, tecnologías y capacidades de apoyo a 
un comercio exterior seguro, (viii) potenciar el uso de infraestructuras existentes para el transporte (marítimo, aéreo y terrestre) y (ix) el establecimiento de alianzas públicoprivadas (APP) que faciliten la interacción sistemática pero efectiva entre los actores ante los constantes cambios del comercio.

Los cambios sugeridos para la ZLC son necesarios y su implementación es casi obligada ya que estos le permitirá adecuarse a la continua transformación que adoptan los mercados a nivel global. Sin ajustes a los actuales mecanismos de funcionamiento, esta zona franca puede caer en la obsolescencia y perder la competitividad ante otros centros similares que se encuentran en la región.

\section{Discusión}

Los resultados muestran una evidente ralentización de la actividad comercial de la ZLC, la cual se acentúa en las importaciones y reexportaciones del primer cuatrimestre del 2020 como un efecto de los shocks de demanda y oferta global ocasionada por la pandemia del COVID-19.

El modelo de distribución vigente bajo el esquema de abastecimiento desde una fuente distante (distant sourcing) ha brindado muchos beneficios a las empresas, particularmente por los bajos costos laborales y otros asociados a la producción. Sin embargo, las fuentes distantes exponen las cadenas de suministros a altos riesgos operacionales y de disrupción de sus flujos (lakovou, Vlachos \& Xanthopoulos, 2010; Christopher et al., 2011). Las recientes distorsiones en las fuentes de abastecimiento originadas en Asia son un ejemplo de cómo la dinámica comercial de la ZLC y el cumplimiento hacia los mercados de la región latinoamericana es afectada.

La propuesta de un nuevo modelo que combina las capacidades existentes y potenciales de la ZLC, las ventajas competitivas del hub logístico de Panamá, más un nuevo enfoque orientado a la transformación, generación de valor agregado y 
distribución de mercancías bajo un esquema de abastecimiento cercano (nearsourcing) permitirá reconvertir a la zona franca en un nodo logístico que potencie su alta conectividad para la distribución regional y la eficiencia en el desarrollo de nuevos procesos productivos.

La implementación de un modelo de abastecimiento cercano para la ZLC es una decisión significativa que retorna la producción a áreas más próximas de los clientes por la cercanía geográfica (Huebsch, 2012; Ferdows, 2009). A su vez, esta alternativa tendrá una influencia de amplio espectro e impacto positivo dentro de la economía del país (Chau, 2013) y representa un fortalecimiento de las cadenas de suministros regionales en el marco de la red global. (Cagliano, De Marco \& Rafele, 2013; Cagliano et al., 2012).

Este nuevo modelo sería complementado por una restructuración general de las cadenas de valor (Hammer \& Plugor, 2016), de la logística de valor agregado local y otras actividades intensivas en el uso de tecnologías y de toma de decisiones estratégicas.

La Zona Libre de Colón representa un importante activo de alto valor para el sistema logístico y la economía panameña, pero su principal actividad basada en el comercio internacional es altamente susceptible a las afectaciones que impactan los centros de producción, la volatilidad de los mercados de consumo final y la fragilidad de las cadenas de suministros.

Se puede inferir que la principal limitante del actual modelo de negocios que muestra fuertes señales de agotamiento es la lenta incorporación de mecanismos que agilicen el comercio, por lo es necesario adoptar cambios estructurales de carácter normativos, institucionales y procedimentales con un fuerte componente tecnológico, de digitalización y flexibilidad comercial. 
Como parte de las nuevas políticas públicas, deben tomarse acciones para la realización de ajustes estratégico de este nuevo modelo de funcionamiento propuesto que permita modernizar la Zona Libre de Colón, expandir su actividad comercial e impulsar su competitividad.

\section{Agradecimiento}

Agradezco al Lic. Daniel Rojas, presidente de la Asociación de Usuarios de la Zona Libre de Colón, por compartir su visión sobre esta zona franca y sus comentarios al borrador de este artículo.

\section{Referencias Bibliográficas}

Araz, O. M., Choi, T. M., Olson, D. L., \& Salman, F. S. (2020). Data Analytics for Operational Risk Management. Decision Sciences. Vol 00(0), April 2020. https://doi.org/10.1111/deci.12443

Banco Interamericano de Desarrollo (BID). (2020a). El impacto del COVID -19 en las economías de la región: Centroamérica, Haití, México, Panamá, República Dominicana. Abril 2020. Disponible en: https://publications.iadb.org/publications/spanish/document/El_impacto_del_C OVID-19_en_las_economías_de_la_región_Centroamérica.pdf

Banco Interamericano de Desarrollo (BID). (2020b). Estimaciones de las tendencias comerciales: América Latina y el Caribe. Edición 2020. 1T. Disponible en: https://publications.iadb.org/es/estimaciones-de-las-tendencias-comercialesamerica-latina-y-el-caribe-edicion-2020-1t

BBCMundo. (2020). Coronavirus en América Latina: 7 gráficos para entender el avance de la pandemia de covid-19 en la región. 27 de abril de 2020. Disponible en: https://www.bbc.com/mundo/noticias-america-latina-52405371

Cagliano, A. C., De Marco, A., \& Rafele, C. (2013). The Impact of Near Sourcing on Global Dynamic Supply Chains: A Case Study. In: Kreowski HJ., Scholz-Reiter B., Thoben KD. (eds) Dynamics in Logistics. Lecture Notes in Logistics, 489- 
498. Springer, Berlin, Heidelberg. https://doi.org/10.1007/978-3-642-35966$8 \_41$

Cagliano, A. C., De Marco, A., Rafele, C. \& Arese, M. (2012). A decision-making approach for investigation the potential effects of near sourcing on supply chain. Strategic Outsourcing: An International Journal, 5(2), 100-120. https://doi.org/10.1108/17538291211257574

Centro Nacional de Competitividad (CNC). (2019). Prospectiva e Inteligencia Competitiva (Síntesis): La Zona Libre de Colón. Disponible en: https://cncpanama.org/cnc/index.php/38-prospectiva-e-inteligenciacompetitiva-de-la-zona-libre-de-colon

Chau, H. M. (2013). The impact of near-sourcing on the port of Rotterdam. Erasmus University Rotterdam. Master thesis. Disponible en: http://citeseerx.ist.psu.edu/viewdoc/download?doi=10.1.1.836.2523\&rep=rep1 \&type=pdf

Clavellina, J.L. \& Domínguez, M.I. (2020). Implicaciones económicas de la pandemia por COVID-19 y opciones de política. Instituto Belisario Domínguez. México. Disponible en: http://www.bibliodigitalibd.senado.gob.mx/handle/123456789/4829

Contraloría General de la República (CGR). (2019). Anuario de Comercio Exterior, Volumen III, Zona Libre de Colón 2017. Publicaciones-Situación Económica. Instituto de Estadística y Censo. Disponible en: https://www.inec.gob.pa/publicaciones/Default3.aspx?ID_PUBLICACION=922 \&ID_CATEGORIA=4\&ID_SUBCATEGORIA=20

Christopher, M., Mena, C., Khan, O.\& Yurt, O. (2011). Approaches to managing global sourcing risk. Supply Chain Management, 16(2), 67-81. https://doi.org/10.1108/13598541111115338

Ferdows, K. (2009). Shaping global operations. Journal of Globalization, Competitiveness \& Governability, 3(1), 136-148. Disponible en: https://www.redalyc.org/pdf/5118/511851320008.pdf 
Flórez, V., Lancheros, L. \& Malambo, M. (2018). Impacto de la logística en Panamá. Tesis de Grado. Universidad del Rosario, Colombia. Disponible en:

Forbes Centroamérica. (2020). Panamá: empresarios recomiendan reabrir la Zona Libre de Colón. 27 de mayo de 2020. Disponible en: https://forbescentroamerica.com/2020/05/27/panama-empresariosrecomiendan-reabrir-la-zona-libre-de-colon/

Gabinete Logístico (2019). Estrategia Logística Nacional de Panamá 2030. Ministerio de la Presidencia, Gobierno de la República de Panamá. Disponible en: https://repository.urosario.edu.co/handle/10336/18807

Georgia Tech Panama Logistics Innovation and Research Center (GTP). (2020). Zona Libre de Colón-Información General. Disponible en: https://logistics.gatech.pa/es/assets/special-economic-zones/colon-free-zone

Gómez-Rudy, C. (2020). Evaluación de la actividad comercial, retos y oportunidades para las empresas de la Zona Libre de Colón: 2019-2020. Informe Ejecutivo presentado a la Asociación de Usuarios de la Zona Libre de Colón. Abril 2020.

Gómez-Rudy, C.M. \& Ortega-Gómez, E. (2016). Análisis exploratorio sobre los niveles de dependencia comercial de la Zona Libre de Colón. Revista Colón Ciencias, Tecnología y Negocios, 3(2), 1-15.

Hammer, N., \& Plugor, R. (2016). Near-sourcing UK apparel: value chain restructuring, productivity and the informal economy. Industrial Relations Journal, 47(5-6), 402-416. https://doi.org/10.1111/irj.12146

Huebsch, R. (2012). How Can Near-sourcing Help a Company Gain a Competitive Edge? Hearst Newspapers. Small Business Index, 2012.

lakovou, E., Vlachos, D. \& Xanthopoulos, A. (2010). A stochastic inventory management model for a dual sourcing supply chain with disruptions. International Journal of Systems Science, 41(3), 315-324. https://doi.org/10.1080/00207720903326894

Ivanov, D. (2020). Predicting the impacts of epidemic outbreaks on global supply chains: A simulation-based analysis on the coronavirus outbreak (COVID- 
19/SARS-CoV-2) case. Transportation Research Part E: Logistics and Transportation Review, 136. https://doi.org/10.1016/j.tre.2020.101922

Organización Mundial de la Salud (OMS). (2020a). Declaración sobre la segunda reunión del Comité de Emergencias del Reglamento Sanitario Internacional acerca (2005) del brote del nuevo coronavirus (2019-nCoV). Declaración del 30 de enero de 2020. Disponible en: https://www.who.int/es/newsroom/detail/30-01-2020-statement-on-the-second-meeting-of-the-internationalhealth-regulations-(2005)-emergency-committee-regarding-the-outbreak-ofnovel-coronavirus-(2019-ncov)

Organización Mundial de la Salud (OMS). (2020b). Alocución de apertura del Director General de la OMS en la rueda de prensa sobre la COVID-19. Declaración del 11 de marzo de 2020. Disponible en: https://www.who.int/es/dg/speeches/detail/who-director-general-s-openingremarks-at-the-media-briefing-on-covid-19---11-march-2020

Slack, B., \& Gouvernal, E. (2016). Container transshipment and logistics in the context of urban economic development. Growth and Change, 47(3), 406-415. https://doi.org/10.1111/grow.12132

Sokol, J.B. (2017). Notas sobre Políticas de Comercio Exterior. ¿Quo Vadis Zona Libre de Colón? Sus problemas y perspectivas. Revista Investigación y Pensamiento Crítico, 5(3), 59-89. https://doi.org/10.37387/ipc.v5i3.75

Worldometer. (2020). Coronavirus cases and deaths. Disponible en: https://www.worldometer.info/coronavirus

Zhang, Y., Xu, J., Li, H., \& Cao, B. (2020). A novel coronavirus (COVID-19) outbreak: a call for action. Chest, 157(4), e99-e101. https://doi:10.1016/j.chest.2020.02.014

Zona Libre de Colón (ZLC). (2020a). Análisis de la Actividad Comercial de abril 2020. Departamento de Estadística y Estudios Económicos. Disponible en: http://www.zolicol.gob.pa/sites/default/files/2020-05/MOVIMIENTO\%20 COME RCIAL\% 20DE\%20ABRIL\% 202020_0.pdf 
Zona Libre de Colón (ZLC). (2020b). Análisis de la Actividad Comercial 2019. Departamento de Estadística y Estudios Económicos. (http://www.zolicol.gob.pa/sites/default/files/2020-02/MOV\%20COM\%20 DICIEMBRE\% 202019.pdf)

Zona Libre de Colón (ZLC). (2019). Compendio Estadística 2014-2018. Departamento de Estadística y Estudios Económicos. Disponible en: (http://www.zolicol.gob.pa/sites/default/files/201905/COMPENDIOESTADISTICO \%202014-2018.pdf) 